\title{
Obesity and disease severity in people with atrial fibrillation: a community-based cross-sectional survey
}

Dimitrios Koutoukidis, Nicholas Jones, Clare J Taylor, Barbara Casadei, Paul Aveyard

Nuffield Department of Primary Care Health Sciences, University of Oxford, Oxford, UK Division of Cardiovascular Medicine, University of Oxford, Oxford, UK

NIHR Oxford Biomedical Research Centre, Oxford, UK

Background: Atrial fibrillation (AF) is the most common sustained cardiac arrythmia and its incidence is strongly linked with obesity. Weight loss may reduce AF severity in relatively young patients with AF and overweight. However, it is unclear whether symptom severity and AF-related quality of life are associated with obesity in the general population of people with AF.

Methods: Ten general practices in central England identified patients with electrocardiogram-confirmed AF. Each person was asked to complete the validated Atrial Fibrillation Severity Scale (AFSS). The AFSS assessed the AF burden scoring on AF-related symptoms, global well-being, as well as total AF burden measured as a combination of duration, frequency, and severity of an irregular heartbeat. Linear regression examined the association of body mass index (BMI) with AF severity adjusting for age, sex, and comorbidities.

Results: Between October 2018 and May 2019, 881 of 1901 (46\%) mailed responses were returned completed. Participants were on average (SD) 74 (10) years old, $64 \%$ were male, $99 \%$ were of white ethnicity. They were taking 5 (3) prescribed medications, had a BMI of $27.5(5.7) \mathrm{kg} / \mathrm{m}^{2}$, and $26 \%$ were affected by obesity. Sixteen percent reported having never experienced an irregular heartbeat. A $5 \mathrm{~kg} / \mathrm{m}^{2}$ higher BMI was associated with a 0.75 (95\% Cl: $0.35-1.15, p<0.001)$ higher symptom score, where 3 points represent a clinically relevant change in state. A $5 \mathrm{~kg} / \mathrm{m}^{2}$ higher $\mathrm{BMI}$ was associated with a $-0.1(95 \% \mathrm{Cl}:-0.2-$ $0.0004, p=0.07$ ) lower global wellbeing score. The coefficient of the total AF burden for a $5 \mathrm{~kg} / \mathrm{m}^{2}$ higher BMI was $0.1(95 \% \mathrm{Cl}:-0.0016-0.6, \mathrm{p}=0.69)$.

Conclusion: BMI was positively associated with AF-related symptom score in the general population of people with AF but not with other constructs of AF. However, the association did not appear to be clinically meaningful. Future research should examine whether excess weight should be addressed in specific subgroups of people with AF for reducing the severity of patient-reported symptoms and improving individuals' quality of life. 